\title{
Efectos de la Actividad Locomotriz Voluntaria Gestacional sobre la Morfología Cardiaca en Crías Murinas
}

\author{
Effects of Gestational Voluntary Locomotor Activity on Cardiac Morphology in Murine Offspring
}

\author{
Ricardo Henríquez ${ }^{1,2}$;Víctor Armijo ${ }^{3}$; Ana Merino ${ }^{3}$; Felipe Nuñez ${ }^{3}$; \\ Elena Marín-Cascales ${ }^{4}$; JacoboA Rubio-Arias ${ }^{5}$ \& ${\text { Carlos } \operatorname{Vargas}^{3}}^{3}$
}

\begin{abstract}
HENRÍQUEZ, R.; ARMIJO, V.; MERINO, A.; NUÑEZ, F.; MARÍN-CASCALES, E.; RUBIO-ARIAS, J. \& VARGAS, C. Efectos de la actividad locomotriz voluntaria gestacional sobre la morfología cardiaca en crías murinas. Int. J. Morphol., 39(1):95-101, 2021.

RESUMEN: El objetivo del presente estudio es analizar los efectos de la actividad locomotriz voluntaria gestacional, como un tipo de entrenamiento físico, sobre la morfología de la bomba cardíaca de la cría, en modelo murino de la cepa CF-1. 12 hembras gestantes fueron divididas aleatoriamente en un grupo control y un grupo que realizó actividad locomotriz voluntaria, accediendo a una rueda de actividad durante los primero 12 días de gestación. Se evaluó la morfología cardiaca mediante cortes transversales, midiendo espesor y área de las paredes del ventrículo derecho, ventrículo izquierdo y septum, tanto en valores absolutos como en valores relativos a la masa corporal del individuo. Se observó que la masa corporal de las crías control (GC) fue significativamente mayor que las del grupo cuyas hembras accedieron a la rueda de actividad (GE) ( $<<0.01)$. Solo hubo diferencias en los valores absolutos de espesores y áreas miocárdicas de ventrículo derecho, entre el grupo GE y GC ( $\mathrm{p}<0.05)$, pero al evaluar los espesores y áreas relativos a la masa corporal se observó que las crías del grupo GE presentaron espesores y áreas significativamente mayores que las que grupo GC ( $\mathrm{p}<0.01$ ). En conclusión, la actividad física gestacional altera el desarrollo morfológico de la bomba cardíaca en ratones CF-1, aumentando significativamente el espesor y área de las paredes miocárdicas en relación a la masa corporal total de la cría.
\end{abstract}

PALABRAS CLAVE: Morfogénesis; Ventrículos cardiacos; Embarazo; Ejercicio.

\section{INTRODUCCIÓN}

Cada vez son más frecuentes las recomendaciones de actividad o entrenamiento físico en etapa gestacional, aludiendo a efectos beneficiosos tanto en la madre como en el individuo gestante (Bahls et al., 2014; Mottola et al., 2019). En la madre se ha descrito que la práctica crónica de actividad física durante el embarazo disminuye el riesgo de diabetes gestacional e hipertensión durante el embarazo (Marcoux et al., 1989; Padayachee \& Coombes, 2015). Además, se ha descrito que el entrenamiento físico gestacional regula la ganancia de peso durante el embarazo (Barakat $e t$ al., 2009). Existe evidencia científica de que el ejercicio físico durante la gestación induce menor prevalencia de cesáreas (Ronnberg et al., 2015) y reduce el riesgo de macrosomías (Ruiz et al., 2013). En modelos animales se ha demostrado que el entrenamiento físico gestacional mejora parámetros fisiológicos metabólicos en las crías, eva- luadas en su etapa de adultez, por ejemplo, se ha observado una mejor regulación de los niveles y la sensibilidad de insulina (Vanheest \& Rodgers, 1997). Sin embargo, no todos los efectos reportados han sido beneficiosos, en modelos animales, se ha observado que niveles moderados o altos de entrenamiento físico gestacional inducen bajo peso al nacer de la cría (Stanford et al., 2015; Sheldon et al., 2016). También se ha encontrado evidencia de aumento de los niveles de actividad física en crías adultas (Eclarinal et al., 2016), asociándose con comportamiento de hiperactividad en humanos.

Existe evidencia que patologías, como las cardiovasculares, tienen su origen en etapas gestacionales, demostrando que el comportamiento materno gestacional y las situaciones experimentadas durante este periodo, influi-

\footnotetext{
${ }^{1}$ Doctoral Program in Sports Science, Faculty of Sport Sciences, Catholic University of Murcia, UCAM, Guadalupe, Murcia, España.

${ }^{2}$ Carrera de Kinesiología, Facultad de Ciencias de las Salud, Universidad Central de Chile, Chile.

${ }^{3}$ Escuela de Kinesiología, Facultad de Medicina, Universidad de Valparaíso, Valparaíso Chile.

${ }^{4}$ Research Center for High Performance Sport, Catholic University of Murcia, UCAM, Guadalupe, Murcia, España.

${ }^{5}$ LFE Research Group, Department of Health and Human Performance, Faculty of Physical Activity and Sport Science-INEF, Universidad Politécnica de Madrid, 28040 Madrid, España.
} 
HENRÍQUEZ, R.; ARMIJO, V.; MERINO, A.; NUÑEZ, F.; MARÍN-CASCALES, E.; RUBIO-ARIAS, J. \& VARGAS, C. Efectos de la actividad locomotriz voluntaria gestacional sobre la morfología cardiaca en crías murinas. Int. J. Morphol., 39(1):95-101, 2021.

rán en el desarrollo del feto, repercutiendo al nacer y probablemente durante gran parte de la vida del individuo (Barker, 2007). En este sentido, May et al. (2016) observaron que el entrenamiento durante la etapa gestacional puede modificar en el gestante la actividad autonómica cardíaca, aumentar la fracción de eyección cardíacaen etapas gestacionales tardías (Brik et al., 2019) e inducir que la cría presente frecuencias cardíacas menores durante la realización deejercicio físico (Roldan-Reoyo et al., 2015). Está bien documentado que todas estas modificaciones funcionales podrán ser adaptativas, dependiendo del contexto en el cual se desarrolle el individuo y estarán directamente influenciadas por la variación de las estructuras anatómicas y morfológicas que comprenden los sistemas, en este caso, el sistema cardiovascular (Barker). No obstante, hasta el momento se desconoce qué efectos morfológicos puede tener el entrenamiento físico sobre la bomba cardíacaen desarrollo y cómose podríanasociar estas posibles modificaciones al contexto funcional.

En base a esta interrogante, el objetivo del presente estudio es analizar los efectos de la actividad locomotriz voluntaria gestacional, como un tipo de entrenamiento físico, sobre la morfología de la bomba cardíacade la cría, en modelo murino de la cepa $\mathrm{CF}-1$. Los resultados de este estudio plantean que el entrenamiento físico gestacional, no solo variaría la funcionalidad del sistema cardiovascular, si no también la morfología del corazón.

\section{MATERIAL Y MÉTODO}

Animales y condiciones de laboratorio: Se realizóun estudio de tipo analítico, experimental, controlado y aleatorizado. Se utilizaron 12ratones hembra de la cepa CF1. Los ratones hembra fueron alojadas de a pares en jaulas plexiglas con ambiente controlado y ciclo luz-oscuridad 12:12 horas. Cada jaula fue aseada 2 veces por semana, manteniendo a los animales con agua y alimento ad libitum. Cabe destacar que todos los protocolos de manejos y cuidado de los animales están acorde a la guía "Regulación del uso y cuidado de Animales en investigación" de la Comisión Nacional de Investigación Científica y Tecnológica, CONICYT (Comisión Nacional de Investigación Científica y Tecnológica, 2014) y el proyecto de estudio fue aprobado por el Comité ético científico de la Universidad Santo Tomás, código 32.18 .

Durante 5 días consecutivos se llevó a cabo el proceso de habituación de todas las hembras incluidas en el estudio. Dichoproceso consistió en traspasar a cada hembra a una jaula individual que incluía una rueda por dos horas diarias, hasta completar los 5 días.
Apareamiento: Posterior al proceso de habituación se alojaron en una misma jaula dos hembras con un macho. El tiempo de apareamiento fue de 7 días y la confirmación de la fecundación fue a través de frotis vaginal sumado a un control de peso corporal durante el periodo gestacional.

Las hembras preñadas fueron separadas aleatoriamente en dos grupos: un grupo control (GC) y un grupo ejercicio (GE). Posteriormente las crías del sexo masculino de las hembras gestantes fueronllamadas como crías GCy crías GE.Se evaluó el peso de la hembra gestante día a día desde el comienzo de la gestación hasta el día previo al parto.

Protocolo de actividad locomotriz voluntaria: Las hembras del GE fueron trasladadas a una jaula individualcon rueda de actividad, a la cual tuvieron acceso durante las 24 horas del día desde el día gestacional 1 al día 11. Se contaron las revoluciones diarias por medio de un sensor electromagnético. A partir del día gestacional 14, a las hembras del GE se les trasladó a una jaula sin acceso a rueda de actividad. Las revoluciones diarias fueron registradas cada 24 horas, expresándose en un promedio de revoluciones por hora/diario.Las hembras del GC se mantuvieron durante todo el periodo gestacional en una jaula individual sin acceso a rueda de actividad.

Sacrificio y tratamiento histológico: Para el presente estudio solo se consideraron las crías macho. Al séptimo día post natal (P7), periodo de crecimiento que es asimilable a los primeros días del nacimiento humano (Ribeiro et al., 1991), las crías fueron pesadas para luego sacrificarlas mediante una inyección de Tiopental intraperitoneal (minimizando el sufrimiento). Después del sacrificio, se diseccionó para extraer el corazón y se seccionó para separar atrio y grandes vasos de ventrículos. Se procedió a fijar postmortem en Bouin (71\% solución saturada de ácido pícrico, $24 \%$ formaldehído y $5 \%$ ácido acético). Posterior a la extracción, las muestras se deshidrataron en una escala de alcoholes crecientes: $70 \%, 96 \%$ y $100 \%$, para luego embeber en xilol y, finalmente, proceder a la impregnación del tejido cardíacocon parafina. A continuación, con un micrótomo Leica RM2235® (Buffalo, USA), se efectuaroncortes histológicos en láminas con un grosor de $5 \mu \mathrm{m}$, para formar y montar las placas. Las muestras fueron rehidratadas y teñidas con HematoxilinaEosina, siguiendo las instrucciones del fabricante para su utilización (Al-Doaiss et al., 2019). Por último, las muestras fueron montadas en placas para su análisis.

Selección de muestra, captura de imagen y medición de la muestra: Las muestras utilizadas fueron las crías machos de las camadas obtenidas correspondientes a los GC y GE. Para medir los parámetros de las zonas se obtuvieron 
microfotografías de cada muestra mediante el modelo de cámara Leica ICC50 W® para microscopio electrónico (Buffalo, USA).

La medición de las muestras se llevó a cabo mediante el Software Image-Pro Plus versión 6.0® para Windows 7® (Rockville, USA).El criterio de inclusión de la microfotografía para la medición de cada una de las variables por separado fue la indemnidad de la ultraestructura en el corte histológico. Se seleccionó y midió un corte cada $30 \mu \mathrm{m}$ hasta abarcar toda la muestra desde base a ápice cardíaco.

Análisis morfológico de la muestra: Para calcular el grosor de pared delVentrículo Derecho (VD), se utilizaron 5mediciones por corte en cada una de las imágenes. En primer lugar, se identificaron en la muestra los extremos del VD y se trazaron dos rectas hacia los márgenes laterales del corazón (VD1 y VD5), ubicándose perpendiculares a la superficie epicárdica. Después, en el punto medio de estas rectas, se trazó la tercera recta hacia el margen lateral del corazón (VD3). Finalmente, en los puntos medios, entre las rectas de los extremos y la recta central, setrazaron 2 rectas más (VD2 y VD4), las cuales también fueronproyectadas hacia el margen lateral del corazón. A cada recta se le evaluóla longitud, promediando la longitud media por corte y por corazón (Fig. 1).

De manera similar a la evaluación del grosor del VD, se evaluó el grosor del ventrículo Izquierdo (VI), trazando 5 rectas perpendiculares a la superficie epicárdica (VI1, VI2, VI3, VI4 y VI5), distribuidas de la misma manera descrita para el ventrículo derecho (Fig. 1).
Para calcular el grosor del septum (S), se utilizaron tres mediciones por corte. Primero se trazaron 2 rectas uniendo los extremos de las cavidades ventriculares derecha e izquierda (S1 y S3), por la zona anterior y posterior, para finalmente trazar una recta en la zona media entre ambos extremos (S2), uniendo las cavidades derecha e izquierda. $\mathrm{Al}$ igual que en las evaluaciones de VI y VD, a cada recta se le evaluó la longitud, promediando la longitud media por corte y luego por corazón (Fig. 1).

Para calcular el área del VI se delimitó un polígono por todo el contorno de VI, desde la recta VI1 hasta la recta VI5. Para el cálculodel área del S, se trazó un polígono por todo el contorno del septum, siendo delimitado por los trazos VI1, VI5, VD1 y VD5. Para calcular el área del VD, se delimitó un polígono por todo el contorno de VD, desde la recta VD1 hasta la recta VD5 (Fig. 1).

Cada órgano fue medido desde su extremo céfalo a caudal, a través del número de cortes histológicos. Teniendo en cuenta que el corte tiene un espesor de $5 \mu \mathrm{m}$, se calculó el tamaño del órgano.

Las evaluaciones morfológicas se presentanen valores absolutos y en valoresnormalizados en base al peso corporal total de la cría, para conocer la relación entre el tamaño de la bomba cardíacay los tejidos periféricos a irrigar.

Peso de la cría y corazón: Luego del sacrificio, todas las crías fueron pesadas en la balanza de laboratorio digital Gram FH - 2000® (Santa Clara, España) para la evaluación de masa de cada cría.

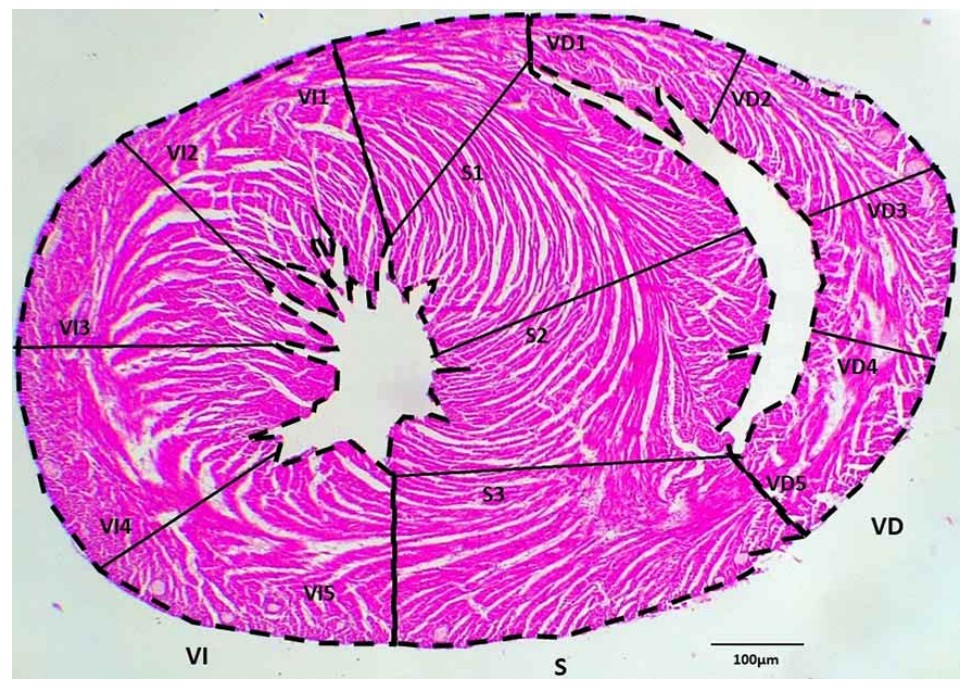

Fig. 1. Distribución de las evaluaciones morfométricas de corte transversal de corazón. Línea entrecortada: polígono para perímetros de áreas; Línea continua: rectas para evaluación de espesor. VD: ventrículo derecho; VI: ventrículo Izquierdo; S: septum. Referencia $100 \mu \mathrm{m}$
Luego del sacrificio, se procedió a diseccionar el corazón de la cría según lo descrito. Todos los órganos fueron evaluados en la balanza digital Gram FH - 2000® (Santa Clara, España).

Análisis Estadístico: Para el análisis estadístico realizado en el presente trabajo, se utilizó el software programa R Studio 1.1.383 (Boston, USA) .Con el fin de analizar el comportamiento de las variables se aplicó el test Shapiro-Wilk, constatando si la muestra es paramétrica. Posterior a esto, se realizó pruebas t de Student en el caso de que las variables provengan de una población con distribución normal y la prueba U de Mann y Whitney en el caso de que las variables no presenten una distribución normal. Se calculó el tamaño del efecto (ES) de la intervención a través de $\mathrm{d}$ de Cohen (d), clasificándolo según lo descrito por Cohen (1992). Se consideró un nivel de significancia de $\mathrm{p}<0,05$. 


\section{RESULTADOS}

Actividad locomotriz voluntaria: Se seleccionaron 12 hembras, las cuales posterior al periodo de apareamiento y el protocolo de habituación fueron divididas de manera aleatoria en un GC y un GE. Las 6 hembras correspondientes al GE fueron alojadas en jaulas con acceso a ruedas de actividad entre el día gestacional 1 (G1) y el día gestacional 11 (G11), durante este periodo se registró la actividad diaria mediante un odómetro magnético, calculando las revoluciones por hora (Rph) promedio diarias (Fig. 2).

Masa corporal: Se seleccionaron al azar 5 crías macho de cada camada, logrando un total de 30 crías de hembras del GC y 30 crías de hembras del GE.La masa corporal de las crías macho seleccionadas de ambos grupos se evaluó al día P7. Las crías del GC tuvieron una masa corporal significativamente mayor que las crías del $\mathrm{GE}(\mathrm{GC}=5,825 \pm$ 0,562 g; GE=4,989 $\pm 0,304$ g; p=0,0012; ES: 0,679) (Fig. 3a).

Masa cardíaca: las crías del GC tuvieron una masa significativamente mayor que las crías del $\mathrm{GE}(\mathrm{GC}=0,025 \pm$ 0,004 g; GE=0,018 $\pm 0,003$ g; p=0,001; ES: 0,703) (Fig. 3b).

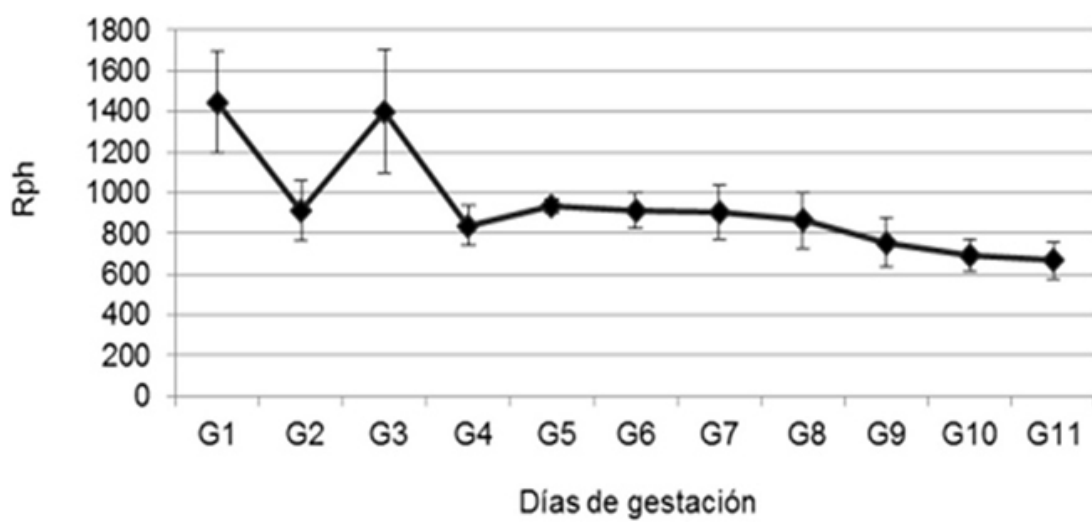

Fig. 2. Registro de las revoluciones por hora (Rph) promedio diarias, entre los días gestacional $1(\mathrm{G} 1)$ y gestacional $11(\mathrm{G} 11)$. Hembras pertenecientes al GE $(\mathrm{n}=6)$. Medida de tendencia central: promedio; barra de dispersión: desviación estándar.
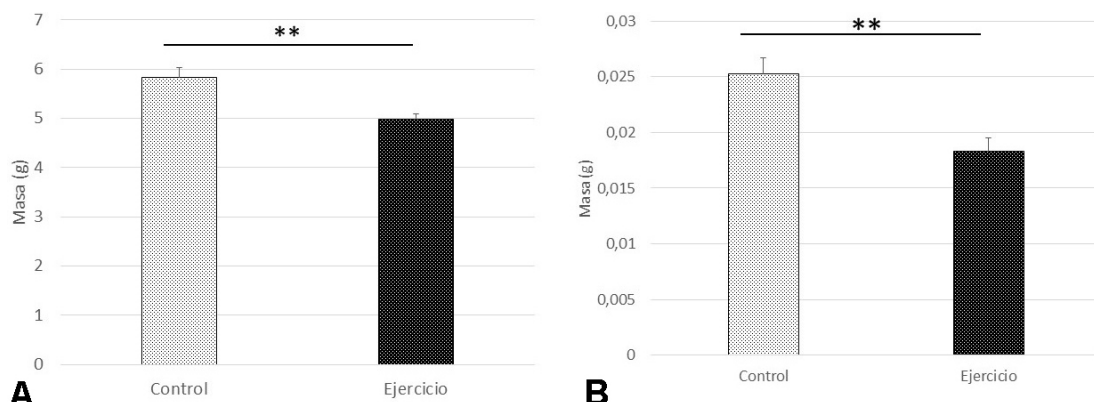

Fig. 3. A. Masa corporal; B. Masa cardíaca. Crías de los grupos GC y GE. n total: 60 crías analizadas. Control $=$ crías de hembras del GC; Ejercicio $=$ crías de hembras del GE $* *$ $=\mathrm{p}<0.01$.

\section{Morfología cardíacaplano transverso:}

- Espesor ventricular: Las crías del GC presentaron un espesor del VD significativamente menor que las crías del GE (Tabla I). En cuanto al espesor del VI y S, no se encontraron diferencias significativas entre ambos grupos (Tabla I).

- Área Ventricular: Las crías del GC presentaron un área de VD significativamente menor que las crías del GE (Tabla I). El área de VI y S no fue significativamente distinta entre ambos grupos (Tabla I).

\section{Morfología en el plano sagital:}

Para analizar la morfología cardíacaen el plano sagital se contabilizó el número de cortes realizados a la estructura cardíaca. Las crías del GC presentaron un número de cortes significativamente mayor que las crías del GE ( $\mathrm{GC}=15,13$ $\pm 2,416$; GE $=12,38 \pm 2,387$; $=0,019$; ES: 0,703).

\section{Morfología cardíaca plano transverso con relación a la masa corporal:}

- Espesor ventricular: Las crías del GC presentaron espesores ventriculares con relación a la masa corporal significativamente menores al GE (Tabla II).

- Área Ventricular: Las crías del GC presentaron áreas ventriculares con relación a la masa corporal significativamente menores al GE (Tabla II). 
Tabla I. Morfología cardíaca plano transverso.

\begin{tabular}{|c|c|c|c|c|c|c|c|}
\hline & & \multicolumn{2}{|c|}{ Control } & \multicolumn{2}{|c|}{ Ejercicio } & \multirow[b]{2}{*}{$\mathrm{P}$ value } & \multirow[b]{2}{*}{ ES } \\
\hline & & Promedio & $\mathrm{DE}$ & Promedio & $\mathrm{DE}$ & & \\
\hline \multirow{3}{*}{ 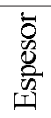 } & $\mathrm{VI}(\mu \mathrm{m})$ & 348,5 & 27,03 & 348,2 & 29,02 & 0,490 & 0,005 \\
\hline & $\mathrm{VD}(\mu \mathrm{m})$ & 131,4 & 18,06 & 139,5 & 11,91 & $0,036^{*}$ & $-0,255$ \\
\hline & $\mathrm{S}(\mu \mathrm{m})$ & 317,2 & 32,85 & 306,5 & 38,97 & 0,322 & 0,146 \\
\hline \multirow{3}{*}{ 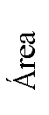 } & $\mathrm{VI}\left(\mu \mathrm{m}^{2}\right)$ & 525821 & 1633,451 & 525855 & 16301,505 & 0,583 & $-0,001$ \\
\hline & $\mathrm{VD}\left(\mu \mathrm{m}^{2}\right)$ & 234852 & 5300,451 & 239838 & 5279,978 & $0,042 *$ & $-0,426$ \\
\hline & $\mathrm{S}\left(\mu \mathrm{m}^{2}\right)$ & 443351 & 13743,881 & 443323 & 13753,323 & 0,413 & 0,001 \\
\hline
\end{tabular}

VI: Ventrículo Izquierdo; VD: Ventrículo derecho; S: Septum; ES: tamaño del efecto. n total= 60 crías. Mann Whitney test. *p $<0,05$.

Tabla II. Morfología cardíaca plano transverso con relación a la masa corporal.

\begin{tabular}{|c|c|c|c|c|c|c|c|}
\hline & & \multicolumn{2}{|c|}{ Control } & \multicolumn{2}{|c|}{ Ejercicio } & \multirow[b]{2}{*}{ P value } & \multirow[b]{2}{*}{ ES } \\
\hline & & Promedio & $\mathrm{DE}$ & Promedio & $\mathrm{DE}$ & & \\
\hline \multirow{3}{*}{ 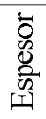 } & $\mathrm{VI} / \mathrm{MC}(\mu \mathrm{m} / \mathrm{g})$ & 59,83 & 1,62 & 69,79 & 1,88 & $0,001 * *$ & $-0,952$ \\
\hline & $\mathrm{VD} / \mathrm{MC}(\mu \mathrm{m} / \mathrm{g})$ & 22,56 & 0,61 & 27,96 & 0,75 & $0,001 * *$ & $-0,969$ \\
\hline & $\mathrm{S} / \mathrm{MC}(\mu \mathrm{m} / \mathrm{g})$ & 54,45 & 1,47 & 61,44 & 1,66 & $0,002 * *$ & $-0,912$ \\
\hline \multirow{3}{*}{$\stackrel{\mathbb{2}}{\stackrel{\mathbb{J}}{2}}$} & $\mathrm{VI} / \mathrm{MC}\left(\mu \mathrm{m}^{2} / \mathrm{g}\right)$ & 90269,699 & 2726,144 & 105402,886 & 3183,167 & $0,005 * *$ & $-0,931$ \\
\hline & $\mathrm{VD} / \mathrm{MC}\left(\mu \mathrm{m}^{2} / \mathrm{g}\right)$ & 40317,939 & 1217,601 & 47071,156 & 1421,548 & $0,003^{* *}$ & $-0,932$ \\
\hline & $\mathrm{S} / \mathrm{MC}\left(\mu \mathrm{m}^{2} / \mathrm{g}\right)$ & 76111,759 & 2298,575 & 88862,096 & 2683,635 & $0,001 * *$ & $-0,928$ \\
\hline
\end{tabular}

VI: Ventrículo Izquierdo; VD: Ventrículo derecho; S: Septum; MC: Masa corporal; ES: tamaño del efecto. n total= 60 crías. Mann Whitney test. $* *=p<0,01$.

\section{DISCUSIÓN}

El objetivo del presente estudio fue analizar los efectos de la actividad locomotriz voluntaria gestacional, como un tipo de entrenamiento físico, sobre la morfología de la bomba cardíaca de la cría en modelo murino de la cepa CF-1. Las hembras de la cepa CF-1 tienen un periodo gestacional de 20 a 21 días, por lo cual su gestación se puede dividir en periodos asimilables a los trimestres de gestación humanos. En el caso de la metodología utilizada en el presente estudio, las hembras gestantes del GE participaron del protocolo de ALV durante las primeras 2 semanas de su periodo gestacional.

En el presente estudio la masacorporal y la masa cardíaca de las crías del GE fue significativamente menor que la de las crías del GC, con un tamaño del efecto clasificado como moderado. Resultados similares han sido obtenidos en el estudio de Oliveira et al. (2004), quienes en un modelo de roedores evaluaron los efectos de entrenamiento físico durante el periodo gestacional en parámetros de masa corporal y renal de las crías. El principal resultado observado fue que las crías de hembras entrenadas durante la gestación presentaron, al nacer,una masacorporal, un tamaño corporal y tamaño renal significativamente menor que las crías del grupo control. Estos resultados fueron explicados por posibles restricciones al flujo sanguíneo placentario registrados durante la gestación. Se ha observadoque entrenamiento físico de alta intensidad pue- de generar restricciones del flujo sanguíneo al feto (Ribeiro et al., 1999), alterando el crecimiento y la ganancia de peso corporal y de los órganos en desarrollo. Estudios en humanos han presentado resultados similares, Hopkins et al. (2010), analizaron los efectos de un programa de entrenamiento en los parámetros del nacimiento, observando que el peso al nacer fue significativamente menor en los hijos de madres entrenadas durante la gestación.

Nuestro estudio es el primero en analizar los efectos de la actividad física gestacional en la morfología cardíaca en las tres dimensiones. En este contexto, se decidió analizar la morfología cardíaca en sus valores absolutos y relacionados con el peso corporal de la cría. El análisis de la morfología cardíacaen relación con la masa corporal total del individuo se realizó porque la bomba cardíaca tiene por función distribuir la sangre por todo el cuerpo, por lo tanto, su función está directamente relacionada con el lecho sanguíneo que se debe irrigar (May et al.). Al analizar la morfología cardíacaen relación con la masa corporal total, observamos que las crías del GE presentan áreas miocárdicas y espesores de sus paredes mayores que las paredes de crías del GC, con un tamaño del efecto clasificado como alto, pero con un menor número de cortes en el plano sagital, lo que supondría un menor tamaño cefalocaudal. Chung et al. (2017) analizaron, en un modelo murino, los efectos del entrenamiento gestacional en las 
células cardíacasde las crías. Los principales resultados observados fueron aumentos significativos en el número de mitocondrias en las células miocárdicas de lascrías de las hembras que entrenaron durante el periodo gestacional. Este aumento mitocondrial estaba acompañado con unincrementode la eficiencia enzimática para generar energía por vía aeróbica. Este cambio celular puede explicar el cambio morfológico cardíaco, ya que las fibras musculares con mayor capacidad aeróbica presentan tamaños mayores que las células con menor capacidad aeróbica. El cambio morfológico también podría ser explicado por aumentos en las concentraciones de factores de crecimiento observados en el miocardio de las crías cuyas hembras realizaron actividad física durante la gestación (Chung et al.). Por otra parte, los resultados obtenidos en el presente estudio concuerdan con los hallazgos funcionales de Brik et al., quienes observaron aumentos en la fracción de eyección cardíaca en crías de hembras entrenadas durante la gestación, pudiendo suponer que las variaciones morfológicas se podrían asociar con variaciones funcionales.

En estudios futuros sería interesante acompañar las evaluaciones morfológicas con análisis fisiológicos, como puede ser el estudio de la actividad eléctrica cardíacao el estudio de las presiones arteriales del individuo. Los presentes resultados,muestran que las crías de hembras con mayor actividad física durante lagestacióntienen una bomba cardíacacon paredes miocárdicas de mayor espesor en relación con los lechos vasculares a irrigar, en comparación a las crías del GC. Por lo tanto, podríamos suponer que la bomba cardíacade las crías del GE podría generar presiones arteriales mayores que las crías del GC.

El presente estudio muestra que la actividad física gestacional altera el desarrollo morfológico de la bomba cardíacaen ratones $\mathrm{CF}$-1, aumentando significativamente el espesor y área de las paredes miocárdicas en relación a la masa corporal total de la cría.

HENRÍQUEZ, R.; ARMIJO, V.; MERINO, A.; NUÑEZ, F.; MARÍN-CASCALES, E.; RUBIO-ARIAS, J. \& VARGAS, C. Effects of gestational voluntary locomotor activity on cardiac morphology in murine offspring. Int. J. Morphol., 39(1):95-101, 2021.

SUMMARY: The objective of the present study is to analyze the effects of gestational voluntary locomotor activity, as a type of physical training, on the morphology of the offspring's heart pump, in a murine model of the CF-1 strain. Twelve (12) pregnant females were randomly divided in a control group and a group performing voluntary locomotor activity, by accessing an activity wheel during the first 12 days of gestation. Cardiac morphology was evaluated using cross sections, measuring thickness and area of the walls of the right ventricle, left ventricle, and septum, both in absolute values and values relative to the individual's body mass. It was observed that the body mass of the control pups (CG) was significantly higher than those of the group whose females accessed the activity wheel (GE) ( $p<0.01)$. Differences were observed only in absolute values of thickness and myocardial areas of the right ventricle, between the GE and GC group ( $\mathrm{p}<0.05$ ). However, when evaluating the thickness and areas relative to body mass, it was observed that the offspring of the GE group presented thicknesses and areas significantly larger than those in the GC group ( $\mathrm{p}<0.01$ ). In conclusion, gestational physical activity alters the morphological development of the heart pump in CF-1 mice, significantly increasing the thickness and area of the myocardial walls in relation to offspring total body mass.

KEY WORDS: Morphogenesis; Heart Ventricles; Pregnancy; Exercise.

\section{REFERENCIAS BIBLIOGRÁFICAS}

Al-Doaiss, A. A.; Ali, D.; Ali, B. A. \& Jarrar, B. M. Renal histological alterations induced by acute exposure of titanium dioxide nanoparticles. Int. J. Morphol., 37(3):1049-57, 2019.

Bahls, M.; Sheldon, R. D.; Taheripour, P.; Clifford, K. A.; Foust, K. B.; Breslin, E. D.; Marchant-Forde, J. N.; Cabot, R. A.; Laughlin, M. H.; Bidwell, C. A.; et al. Mother's exercise during pregnancy programmes vasomotor function in adult offspring. Exp. Physiol., 99(1):20519,2014.

Barakat, R.; Ruiz, J. R.; Stirling, J. R.; Zakynthinaki, M. \& Lucia, A. Type of delivery is not affected by light resistance and toning exercise training during pregnancy: a randomized controlled trial. Am. J. Obstet. Gynecol., 201(6):590.e1-6, 2009.

Barker, D. J. P. The origins of the developmental origins theory. J. Intern. Med., 261(5):412-7, 2007.

Brik, M.; Fernández-Buhigas, I.; Martin-Arias, A.; Vargas-Terrones, M.; Barakat, R. \& Santacruz, B. Does exercise during pregnancy impact on maternal weight gain and fetal cardiac function? A randomized controlled trial. Ultrasound Obstet. Gynecol., 53(5):583-9, 2019.

Chung, E.; Joiner, H. E.; Skelton, T.; Looten, K. D.; Manczak, M. \& Reddy, P. H. Maternal exercise upregulates mitochondrial gene expression and increases enzyme activity of fetal mouse hearts Physiol. Rep., 5(5):e13184, 2017.

Cohen, J. A power primer. Psychol. Bull., 112(1):155-9, 1992.

Comisión Nacional de Investigación Científica y Tecnológica (CONICYT). Regulación del Uso y Cuidado de Animales en Investigación. Santiago de Chile, Comisión Nacional de Investigación Científica y Tecnológica (CONICYT), 2014.

Eclarinal, J. D.; Zhu, S.; Baker, M. S.; Piyarathna, D. B.; Coarfa, C.; Fiorotto, M. L. \& Waterland, R. A. Maternal exercise during pregnancy promotes physical activity in adult offspring. FASEB J., 30(7):2541-8, 2016.

Hopkins, S. A.; Baldi, J. C.; Cutfield, W. S.; McCowan, L. \& Hofman, P. L. Exercise training in pregnancy reduces offspring size without changes in maternal insulin sensitivity. J. Clin. Endocrinol. Metab., 95(5):2080-8, 2010.

Marcoux, S.; Brisson, J. \& Fabia, J. The effect of leisure time physical activity on the risk of pre-eclampsia and gestational hypertension. $J$. Epidemiol. Community Health, 43(2):147-52, 1989.

May, L. E.; Allen, J. J. B. \& Gustafson, K. M. Fetal and maternal cardiac responses to physical activity and exercise during pregnancy. Early Hum. Dev., 94:49-52, 2016. 
Mottola, M. F.; Davenport, M. H.; Ruchat, S. M.; Davies, G. A.; Poitras, V. J.; Gray, C. E.; Jaramillo Garcia, A.; Barrowman, N.; Adamo, K. B.; Duggan, M.; et al. 2019 Canadian guideline for physical activity throughout pregnancy. Br. J. Sports Med., 52(21):1339-46, 2019.

Oliveira, A. O.; Fileto, C. \& Melis, M. S. Effect of strenuous maternal exercise before and during pregnancy on rat progeny renal function. Braz. J. Med. Biol. Res., 37(6):907-11, 2004.

Padayachee, C. \& Coombes, J. S. Exercise guidelines for gestational diabetes mellitus. World J. Diabetes, 6(8):1033-44, 2015.

Roldan-Reoyo, O.; Pelaez, M.; May, L. \& Barakat, R. Influence of maternal physical exercise on fetal and maternal heart rate responses. Ger. J. Exerc. Sport Res., 49:446-53, 2019.

Ronnberg, A. K.; Ostlund, I.; Fadl, H.; Gottvall, T. \& Nilsson, K. Intervention during pregnancy to reduce excessive gestational weight gain-a randomised controlled trial. BJOG, 122(4):537-44, 2015.

Ruiz, J. R.; Perales, M.; Pelaez, M.; Lopez, C.; Lucia, A. \& Barakat, R. Supervised exercise-based intervention to prevent excessive gestational weight gain: a randomized controlled trial. Mayo Clin. Proc., 88(12):1388-97, 2013.

Sheldon, R. D.; Blaize, A. N.; Fletcher, J. A.; Pearson, K. J.; Donkin, S. S.; Newcomer, S. C. \& Rector, R. S. Gestational exercise protects adult male offspring from high-fat diet-induced hepatic steatosis. J. Hepatol., 64(1):171-8, 2016.

Stanford, K. I.; Lee, M. Y.; Getchell, K. M.; So, K.; Hirshman, M. F. \& Goodyear, L. J. Exercise before and during pregnancy prevents the deleterious effects of maternal high-fat feeding on metabolic health of male offspring. Diabetes, 64(2):427-33, 2015.

Vanheest, J. L. \& Rodgers, C. D. Effects of exercise in diabetic rats before and during gestation on maternal and neonatal outcomes. Am. J. Physiol., 273(4):E727-33, 1997.

\author{
Corresponding author: \\ Ricardo Andrés Henríquez Flores \\ Lord Cochrane 417 \\ Santiago Centro \\ CHILE
}

Email: r.henriquez.f@gmail.com

Received: 03-08-2020

Accepted: 21-09-2020 